\title{
Nicotine dependence treatment: provision of a dedicated programme by the National Addictions Management Service
}

\author{
Lambert Tchern Kuang Low ${ }^{1}$, MRCPsych, MBBS, Charis Wei Ling $\underline{N g}^{1}$, MPH, Cheng $\underline{L e}^{1}$, MBBS, MMed
}

\begin{abstract}
INTRODUCTION
While healthcare efforts in Singapore have lately been focused on the prevention and treatment of diabetes mellitus, a small but important national centre has been working on another important issue: smoking. Smoking is perhaps the top cause of death among lifestyle habits and continues to affect more than one billion people around the world. ${ }^{(1)} 13.3 \%$ of the adult population in Singapore smoke, a prevalence that has remained relatively constant from $13.6 \%$ in 2007.(2) A recent landmark meta-analysis concluded that even one stick of cigarette a day contributes to a substantially increased risk of cardiovascular morbidity. ${ }^{(3)}$ Hence, even if not all recorded smokers are heavy smokers, the healthcare burden can be quite substantial.
\end{abstract}

\section{NICOTINE, THE SOURCE OF ADDICTION}

Nicotine is a highly addictive chemical due to its characteristics: it readily crosses the blood brain barrier and has a quick onset of action with a short half-life. This chemical in cigarette smoke sustains the addiction to cigarettes, while the inhalation route further facilitates the direct delivery of nicotine to the brain. Over time, tolerance to nicotine develops, resulting in unpleasant withdrawal symptoms when plasma nicotine levels fall. ${ }^{(4)}$

Other agents apart from cigarettes carry nicotine and tobacco. In the local context, beedi, shisha (banned in November 2014) and cigars are other modes of smoking. All these methods deliver nicotine, with cigarettes in particular having the risk of delivering more than 7,000 other chemicals, at least 69 of which are known carcinogens. ${ }^{(5)}$ Why then do people continue to smoke despite such health risks? The answer pertains to the nicotine addiction that hijacks the reward centres of the brain. Once an addiction is formed, it becomes infinitely much harder to quit any habit. The brain's neurochemical pathways are rewired and help is therefore needed to fight the addiction. ${ }^{(6)}$

\section{MODEL OF SMOKING CESSATION TREATMENT}

The gold standard for smoking cessation is currently a combination of pharmacological support such as nicotine replacement therapies (NRT) (i.e. patches, gum, lozenges, tablets or inhalers), sprays, bupropion or varenicline, together with behavioural approaches such as counselling. ${ }^{(7)}$ Based on the Health
Promotion Board's 2013 guidelines on smoking cessation, multiple person-to-person behavioural support sessions such as counselling are recommended, as well as telephone follow-up..$^{\left({ }^{8}\right)} \mathrm{A}$ combination of behavioural support and medication can increase the chances of successfully quitting by $70 \%-100 \%$ compared to usual care, brief advice or less intensive behavioural support. ${ }^{(7)}$

Through a combination of NRT or medications with behavioural approaches, any attempt to quit has a $19.0 \%-55.2 \%$ rate of success at the end of one year. ${ }^{(9-12)}$ Another study estimated the effectiveness of various pharmacologic therapies combined with behavioural therapy on smoking cessation to be $23.0 \%$ (bupropion), 32.5\% (varenicline) and 52.8\% (NRT), with an overall success rate of $35.0 \% .^{(13)}$

\section{SMOKING CESSATION SERVICE BY NATIONAL ADDICTIONS MANAGEMENT SERVICE}

In March 2017, the National Addictions Management Service of the Institute of Mental Health (IMH) initiated a smoking cessation service that combined behavioural approaches and medication treatment. At the initial assessment, the attending doctor assesses whether the patient is dependent on nicotine and the severity of dependence based on the Fagerstrom Test for Nicotine Dependence (FTND) questionnaire. ${ }^{(14)}$ Thereafter, the level of expired carbon monoxide $(\mathrm{CO})$ from the patient is measured through the piCO ${ }^{\text {TM }}$ Smokerlyzer ${ }^{\circledR} .{ }^{(15)} \mathrm{CO}$ can remain in the bloodstream for up to 24 hours and is measured in parts per million (ppm). ${ }^{(16)}$ The piCO Smokerlyzer defines $\mathrm{CO}$ levels in non-smokers as 0-6 ppm, low-dependence smokers as 7-15 ppm and strongly addicted smokers as over 15 ppm. ${ }^{(16)}$

This is followed by a thorough clinical history-taking and physical assessment. The clinical history focuses on elucidating the chronicity of the smoking; the number of cigarettes smoked per day; any history of quit attempts; any withdrawal symptoms during previous quit attempts; any previous treatment for smoking cessation; triggers for smoking; the presence of any physical and psychiatric ailments; the presence of any contraindications to any of the proposed treatment options; and the pattern of smoking, starting with the time to the first cigarette. This assessment guides the therapeutic intervention. The patient's financial and lifestyle considerations are also

${ }^{1}$ National Addictions Management Service, Institute of Mental Health, Singapore

Correspondence: Dr Lambert Low Tchern Kuang, Consultant, National Addictions Management Service, Institute of Mental Health, Buangkok Green Medical Park, 10 Buangkok View, Singapore 539747. lambert_tk_low@imh.com.sg 
taken into account owing to differences in the treatment cost of the various medications.

The three available medication treatment options are (a) NRT, (b) bupropion and (c) varenicline. Each pharmacological treatment is combined with individual counselling to achieve optimal results. Treatment is individualised to the particular individual based on demographics, individual preferences, cost considerations, and history of chronic medical or psychiatric ailments. This programme is separate from the Health Promotion Board's 'I Quit' smoking cessation counselling programme. When choosing a medication option, the patient's lifestyle should be taken into consideration. For example, it may be inappropriate for patients of certain professions to be seen chewing nicotine gum. For those who perspire a lot during work, it is difficult to use a nicotine patch.

If NRT is chosen as the modality of treatment, its amount is titrated according to the current levels of smoking. It is recommended that both a short- and a long-acting nicotine replacement product are used simultaneously. The long-acting nicotine product (patch) helps in relieving baseline cravings for nicotine, while the short-acting product (gum/lozenge) helps with breakthrough cravings. The patient is also educated on the proper use of the nicotine gum, as frequent misuse results in the swallowing of nicotine rather than absorption through the buccal mucosa. The most important aspect of NRT is the need for accurate dosing, as underdosing results in treatment failure. The practical aspect of cost also matters; optimal dosage sometimes cannot be instituted due to the high cost of NRT. Hence, when patients are very heavy smokers, it is more economical to consider bupropion or varenicline. Furthermore, NRT is not as effective in women smokers due to their more rapid metabolism of nicotine. ${ }^{(17)}$

Titration of bupropion or varenicline treatment is relatively straightforward, as it is not dependent on the patient's level of smoking. The usual dose for bupropion is $150 \mathrm{mg}$ once a day for three days, increasing to $150 \mathrm{mg}$ twice a day and continued for 7-12 weeks. ${ }^{(18)}$ The standard regimen for varenicline is $0.5 \mathrm{mg}$ per day for the first three days, followed by $0.5 \mathrm{mg}$ twice daily for four days and $1.0 \mathrm{mg}$ twice daily from Day 8 onwards. ${ }^{(13)}$ However, as these are pharmacological agents, there is a potential for untoward side effects and drug-drug interactions. This is different from NRT, in which the active ingredient nicotine is already found in cigarette smoke. Hence, bupropion may not be suitable for patients with a history of seizures, eating disorders and certain psychiatric ailments. Similar considerations exist for varenicline. Between the two treatments, bupropion is very much cheaper, as generic formulations are already available. However, success rates for smoking cessation are better for varenicline. ${ }^{(18)}$

Counselling is part of our treatment process and is aimed at behavioural change. Our counsellors are trained in motivational interviewing techniques to help patients with addictions. The number of individualised sessions to be conducted depends on the progress of the patient. Each session focuses on different objectives such as strengthening commitment to change, stress management and lifestyle changes, reviewing progress and motivational enhancement therapy, and reinforcing the benefits of not smoking. Patients are scheduled to be seen by their attending doctor every month. For each review visit, we work on progressive improvement using modest goals, for example, a 50\% reduction in the level of smoking for heavy smokers compared with the last session. This is opposed to the current approach elsewhere, whereby patients do not usually have any follow-up sessions after a brief consultation with a pharmacist. We have learnt that a consistent, steady approach is more effective than a pressing need for immediate abstinence.

\section{LESSONS LEARNT}

After more than 1.5 years of running this service with referrals streaming in from polyclinics and restructured hospitals, a few lessons have been learnt. We have seen 94 patients since starting this service in March 2017. The majority were male (84.0\%) and aged 15-74 years. In terms of ethnicity, $59.6 \%$ were Chinese, $20.2 \%$ were Indian and $17.0 \%$ were Malay. 35 (37.2\%) patients had been diagnosed with psychiatric comorbidities, while 49 $(52.1 \%)$ of them had physical illnesses. Smoking duration ranged from 10 months to 58 years. About 20.0\% were first-timers to quitting smoking, and $74.5 \%$ had never sought treatment for smoking previously. More than one-third (35.1\%) of the patients smoked $\leq 10$ cigarettes per day, another $43.6 \%$ smoked 11-20 cigarettes a day, while the remaining $21.3 \%$ smoked $>20$ cigarettes a day. Based on the FTND, 44.7\%, 42.6\% and $12.7 \%$ had low, moderate and high nicotine dependence, respectively.

The first lesson is that consistent follow-up is key to treatment success. The second lesson is that having a visual aid (i.e. the Smokerlyzer) to help smokers monitor their improvement is important. This gives them a psychological tether and a yearning to do better at the next session. Lastly, it is important to individualise treatment based on the individual's lifestyle and cost considerations. Among our 94 patients, most opted for NRT (41.5\%), followed by bupropion (20.2\%) and varenicline (14.9\%). Over the course of treatment, we sometimes had to switch from one modality to another or even combine modalities because the first treatment had not worked or treatment effects had plateaued. Often, good outcomes could be reached even though the initial treatment had not worked. After excluding 23 patients who were not due for their three-month follow-up, we found that 49 $(69.0 \%)$ of the remaining 71 patients defaulted on their follow-up appointment. Of the 22 patients who completed the three-month follow-up, 6 (27.3\%) stopped smoking completely, while another $13(59.1 \%)$ reduced the number of cigarettes smoked. This is comparable with the other local smoking cessation services with published outcomes. ${ }^{(19-21)}$

Currently, we are in the process of extending smoking cessation services to the whole of $\mathrm{IMH}$, as smoking is quite prevalent among patients with mental illness (PMIs) and contributes to the increased morbidity and mortality of our patients. We hope that the same approach would be effective. With the worldwide prevalence of smoking in PMls being disproportionately higher than that in the general population, ${ }^{(22-23)}$ it is important to lower the health inequality experienced as a result of smoking. 


\section{REFERENCES}

1. National Registry of Diseases Office \& Substance Abuse Department, Health Promotion Board, Singapore. World No Tobacco Day Information Paper. Available at: https://www.nrdo.gov.sg/docs/librariesprovider3/default document-library/nrdo-wntd-info-paper-2015.pdf. Accessed May 23, 2018.

2. Khoo EKH. Youth must know link between smoking, disease. TODAY 2015 Jun 4. Available at: https://www.todayonline.com/voices/youth-must-knowlink-between-smoking-disease/. Accessed May 23, 2018.

3. Hackshaw A, Morris JK, Boniface S, Tang JL, Milenković D. Low cigarette consumption and risk of coronary heart disease and stroke: meta-analysis of 141 cohort studies in 55 study reports. BMJ 2018; 360:j5855.

4. Benowitz NL. Pharmacology of nicotine: addiction, smoking-induced disease, and therapeutics. Annu Rev Pharmacol Toxicol 2009; 49:57-71.

5. Mendel JR, Baig SA, Hall MG, et al. Brand switching and toxic chemicals in cigarette smoke: a national study. PLoS One 2018; 13:e0189928.

6. De Biasi M, Dani JA. Reward, addiction, withdrawal to nicotine. Ann Rev Neurosci 2011; 34:105-30.

7. Stead LF, Koilpillai P, Fanshawe TR, Lancaster T. Combined pharmacotherapy and behavioural interventions for smoking cessation. Cochrane Database Sys Rev 2016; (3):CD008286.

8. Health Promotion Board, Singapore. Treating Tobacco Use and Dependence. HPB-MOH Clinical Practice Guidelines 1/2013. Available at: https://www.hpb. gov.sg/docs/default-source/pdf/hpb-moh-clinical-practice-guidelines-treatingtobacco-use-and-dependence-2013.pdf. Accessed May 23, 2018.

9. Abdullah AS, Hedley AJ, Chan SS, Ho WW, Lam TH. Establishment and evaluation of a smoking cessation clinic in Hong Kong: a model for the future service provider. J Public Health (Oxf) 2004; 26:239-44.

10. Hsueh KC, Chen CY, Yang YH, Huang CL. Smoking cessation program in outpatient clinics of Family Medicine Department in Taiwan: a longitudinal evaluation. Eval Health Prof 2010; 33:12-25.

11. Solak ZA, Telli CG, Erdinc E. Results of smoking cessation program. Turk Toraks
Derg 2003; 4:73-7.

12. Wood-Baker R. Outcome of a smoking cessation programme run in a routine hospital setting. Intern Med J 2002; 32:24-8

13. Yilmazel Ucar $\mathrm{E}$, Araz O, Yilmaz $\mathrm{N}$, et al. Effectiveness of pharmacologic therapies on smoking cessation success: three years results of a smoking cessation clinic. Multidiscip Respir Med 2014; 9:9.

14. Heatherton TF, Kozlowski LT, Frecker RC, Fagerström KO. The Fagerström Test for Nicotine Dependence: a revision of the Fagerström Tolerance Questionnaire. Br J Addict 1991; 86:1119-27.

15. Bedfont Scientific Ltd. piCO: breath CO monitor to help stop smoking. Bedfont Scientific Ltd, 2018. Available at: https://www.bedfont.com/shop/smokerlyzer/ pico. Accessed May 23, 2018

16. Bedfont Scientific Ltd. piCO+ Smokerlyzer Operating Manual. Issue 14 - June 2014 ed: Bedfont Scientific Ltd; 2014. Available at: https://www.bedfont.com/ file.php?f=ZmlsZSMjNzE0. Accessed May 24, 2018.

17. Mendelsohn C. Women who smoke - a review of the evidence. Aust Fam Physician 2011; 40:403-7.

18. Cahill K, Stevens S, Perera R, Lancaster T. Pharmacological interventions for smoking cessation: an overview and network meta-analysis. Cochrane Database Syst Rev 2013; (5):CD009329.

19. Cheng HM, Liu WC, Chua G, et al. Impact of a pharmacy-led smoking cessation clinic in a dermatology centre. Singapore Med J 2019; 60:31-3.

20. Kng KK, Lauw XT, Tan AS, Earnest A. Effectiveness of smoking cessation services in Tan Tock Seng Hospital, Singapore. Ann Acad Med Singapore 2012; 41:230-2.

21. Zow HC, Hsu AA, Eng PC. Smoking cessation programme: the Singapore General Hospital experience. Singapore Med J 2004; 45:430-4.

22. Annamalai A, Singh N, O'Malley SS. Smoking use and cessation among people with serious mental illness. Yale J Biol Med 2015; 88:271-7.

23. Cook BL, Wayne GF, Kafali EN, et al. Trends in smoking among adults with mental illness and association between mental health treatment and smoking cessation. JAMA 2014; 311:172-82. 